\title{
Building the NinAPro Database: A Resource for the Biorobotics Community
}

\author{
Manfredo Atzori, Arjan Gijsberts, Simone Heynen, Anne-Gabrielle Mittaz Hager, Olivier Deriaz, \\ Patrick van der Smagt, Claudio Castellini, Barbara Caputo, and Henning Müller
}

\begin{abstract}
This paper is about (self-powered) advanced hand prosthetics and their control via surface electromyography (sEMG). We hereby introduce to the biorobotics community the first version of the NINAPRO database, containing kinematic and sEMG data from the upper limbs of 27 intact subjects while performing 52 finger, hand and wrist movements of interest. The setup and experimental protocol are distilled from existing literature and thoroughly described; the data are then analysed and the results are discussed. In particular, it is clear that standard analysis techniques are no longer enough when so many subjects and movements are taken into account. The database is publicly available to download in standard ASCII format.

The database is an ongoing work lasting several years, which is planned to contain data from more than 100 intact subjects and 50 trans-radial amputees; characteristics of the amputations, phantom limbs and prosthesis usage will be stored. We therefore hope that it will constitute a standard, widely accepted benchmark for all novel myoelectric hand prosthesis control methods, as well as a fundamental tool to deliver insight into the needs of trans-radial amputees.
\end{abstract}

\section{INTRODUCTION}

The daily life of hand amputees can be poor compared to what it was before the amputation. Despite the advances in hand mechatronics realised by the rehabilitation / biorobotics community, state-of-the-art self-powered hand prostheses do not as yet offer more than three degrees of freedom over hand and wrist, bulky and costly devices, and very coarse control of the force. Patients control the prosthesis via electromyography recorded using surface electrodes (sEMG) after a long and difficult learning process; for this reason only a limited set of simplified movements (e.g., opening and closing) can be achieved. As a result, $30 \%$ to $50 \%$ of the amputees do not use their prosthesis regularly [1].

The research community has been working vigorously to increase the level of control in hand prosthetics. The mainstream approach considers a control schema where a classifier is coupled with a regressor. While the classifier is

M. Atzori and H. Müller are with the Department of Business Information Systems at the University of Applied Sciences Western Switzerland (HESSO Valais), Sierre, Switzerland.

C. Castellini and P. van der Smagt are with the Institute of Robotics and Mechatronics of the DLR - German Aerospace Research Center, Wessling, Germany.

B. Caputo and A. Gijsberts are with the Institute de Recherche Idiap, Martigny, Switzerland.

A.-G. Mittaz Hager and S. Heynen are with the Department of Physical Therapy at the University of Applied Sciences Western Switzerland (HESSO Valais), Leukerbad, Switzerland.

$O$. Deriaz is with the Institut de recherche en réadaptation, Service de recherche et contrôle qualité médicale, Clinique romande de réadaptation, Suvacare, Sion, Switzerland. used to understand which hand or grasp posture is desired (e.g., fully stretched hand, power grasp, pointing index), the regressor predicts the force required in the case of a grasping posture. The approaches presented so far in the literature use various techniques for preprocessing the data [2], [3], classifying the postures [3]-[5] and predicting the associated force [3], [6], [7]; despite the differences, all these studies share a common validation procedure, in which experiments are conducted on a proprietary database containing between five and ten intact subjects and amputees, which are executing up to ten different grasp actions, enacting static hand postures or moving their fingers and wrist. (We will from now on collectively denote these actions movements of interest; see section I-A for a more detailed review).

And yet, since none of these data collections are publicly available, direct comparison between various methods can be done only qualitatively and up to a certain extent. As opposed to this, the importance of solid benchmarking protocols and publicly available databases has been confirmed repeatedly, e.g., in the machine vision and image analysis communities [8], [9], where the consensus is that it promotes comparison between methods and pushes forward progress. In addition to this, in the studies presented so far the number of considered movements is typically quite far from the dexterity of a human hand or from what a patient would need in daily life; at the same time, acquiring data only from few subjects makes it difficult to evaluate the generality of the obtained results across gender, age, characteristics of the amputation, etc.

Our aim is to provide the biorobotics community with such a comparison and benchmarking tool, as far as hand prosthetics is concerned. Specifically, the contribution of this paper is the first version of the NINAPRO database, a database of sEMG and kinematic data gathered from 27 intact subjects while performing 52 movements of interest. Upon acceptance of this paper, the NINAPRO database will be made publicly available to the community together with other resources of interest, such as a description of the experimental protocol and acquisition setup, results obtained on the data and so on. ${ }^{1}$

We then perform a preliminary analysis on the database employing a standard machine learning method, showing that this vast increase in the number of movements and subjects with respect to prior work leads to challenging issues in terms

\footnotetext{
${ }^{1}$ The database is temporarily available for the reviewers of this paper at http://ninapro.hevs.ch, login "reviewer", password "reweiver".
} 
of classification accuracy and generality across subjects.

The paper is organised as follows: after reviewing related work we describe the acquisition setup and experimental protocol (Section II) and the analysis along with the results (Section III). We then draw some conclusions and outline the future work (Section IV).

\section{A. Related Work}

The clearest statement of the problem of controlling an advanced hand prosthesis (AHP) using sEMG is probably the one given in [10]. Surface EMG is collected from a subject's forearm skin while performing a number of movements of interest, or producing force patterns of interest; at the same time kinematic (and, when possible, dynamic) data are collected from the upper limb. By kinematic data we hereby mean positions of the fingers, hand and wrist joints, while dynamic data refer to forces exerted at the fingertips or as a whole during, e.g., grasping. While intact subjects are examined by recording sEMG and kinematic data from the same arm, in the case of amputees at least three strategies have been developed and successfully tested (see, e.g., [3], [11]), each consisting of recording sEMG from a stump, and kinematic and dynamic data from an intact limb, while eliciting movements of interest either by imitation or bilateral coordinated motion.

The signals are then preprocessed, features are extracted from them, and the resulting data are used to train a supervised machine learning method. The learned association between sEMG and hand posture, position, or force can later on be used to control the AHP. Notice that sEMG is related to forces rather than to positions, but nevertheless a large corpus of research has successfully dealt with mapping sEMG to kinematic features of the hand (e.g., finger movement [12], [13] and grasping postures). The success is due to the low inertia of the hand joints, thanks to which a correspondence between isotonic and isometric hand muscle configurations and positions can be established. (Attempts at mapping sEMG to whole arm position have been successfully made [14]-[17], which makes this statement even stronger.) The first example of this technique is probably [18], dating back to 45 years ago. The advancements since then have been impressive: according to two recent surveys [19], [20], up to twelve hand postures have been classified with accuracy rates between $80 \%, 90 \%$ and more [13], [21]. Analyses on up to twelve intact subjects [13] and up to six amputees (both trans-radials and trans-humerals) are reported.

Notwithstanding this, to the best of our knowledge there is still no widely accepted, standard protocol to gather data in this kind of experiments, although remarkable guidelines for sEMG processing are indeed available [10], [22]-[24]. Similarly, there is no consensus either about the type of data filtering, processing, and storing, despite a wide range of feature extraction and machine learning methods that have been considered for this setting (see the surveys again). Even worse, there is no publicly available database so far, in which both intact subjects and amputees are classified according to their clinical history. As a result, each research group chooses a different way to record, store, and process the data, while the few experiments performed on amputees (among which [3], [13], [25], [26]) are only conclusive for the (small) set of subjects considered in the corresponding study.

\section{BUILDING THE DATABASE}

\section{A. Acquisition Setup}

1) Kinematic Data: A 22-sensor Cyberglove II dataglove ${ }^{2}$ (see Figure 1, right panel) is used to gather the finger positions. (The setup includes both a left- and a righthanded glove, in order to accommodate for both-sides transradial amputees.) The Cyberglove is a light fabric, rather elastic glove, onto which 22 strain gauges are sewn; the sewing sheaths are chosen carefully by the manufacturer, so that the gauges exhibit a resistance that is proportional to the angles between pairs of hand joints of interest, including inter-phalanx, metacarpum-phalanx, inter-finger adduction/abduction and palm arch angles. The device represents these 22 joint angles as 8-bit values, for an average resolution of less than one degree, depending on the size of the subject's hand, proper wearing of the glove, and the rotation range of the considered joint. (For practical reasons the subject must wear a cotton glove below the Cyberglove; an initial round of data gathering revealed that this would not limit the precision of the device.) The Cyberglove is nowadays a standard hand posture collection device in virtual reality, clinical and academic envinronments. In addition to the Cyberglove, a standard commercially available 2-axis inclinometer is fixed onto the subject's wrist and used to collect the wrist orientation.

2) Surface EMG: Muscular activity is gathered using ten active double-differential OttoBock MyoBock 13E200 surface EMG electrodes ${ }^{3}$, which provide an amplified, bandpass-filtered and rectified version of the raw SEMG signal. Whereas the raw sEMG signal has a bandwith of $15-500 \mathrm{~Hz}$, the bandwidth of this signal is in the range $0-25 \mathrm{~Hz}$. The electrodes are gifted with an amplification gauge ranging from 2,000 to 100,000 times. Qualitative experiments revealed that a safe setting for the amplification gauge was in the middle of the range, corresponding to about 14,000 times [27]. These electrodes are commonly reported in literature as being both reliable and safe. Their use requires no cleaning or shaving, making the acquisition process more comfortable both for the experimenter and for the subject. As far as the placement of the electrodes is concerned, several attempts at finding the right forearm muscles on healthy subjects exist (see, e.g., [24], [28]), based upon anatomic guidelines and basic considerations on the structure of a muscle and the electric field it produces when moved voluntarily. Unfortunately, these attempts have little or no meaning when dealing with amputees due to the uniqueness of each amputation. In [13], placement of up to 32 electrodes is described (and used) based on the severity

\footnotetext{
${ }^{2}$ Cyberglove Systems LLC, http://www.cyberglovesystems. $\mathrm{com}$

${ }^{3}$ Otto Bock HealthCare GmbH, http://www.ottobock.com
} 
of the amputation. Notwithstanding this, early research on pattern matching for sEMG [2], [29], recently confirmed in [3], [13], prove that targeted placement of electrodes is not required as pattern recognition techniques can compensate for suboptimal placement, or even take advantage of muscle cross-talk. In order to take advantage of both approaches, eight uniformly spaced electrodes are placed just beneath the elbow at a fixed distance from the radio-humeral joint, while two more are placed on the flexor and extensor muscles (see Figure 1). In the case of amputees, it must be checked for each single case whether the placement of the two targeted electrodes makes sense.

\section{B. Experimental Protocol}

1) Preliminaries: The experiment was approved by the Ethics Commission of the state of Valais (Switzerland), main place for data acquisition. Safety of the involved hardware and adherence to the WMA (World Medical Association) Declaration of Helsinki are enforced. Informed consent as well as personal and clinical data are obtained in written form from each subject prior to the start of the experiment. This data includes age, gender, height, weight, fitness, laterality, and self-reported health status. In case of amputees, we also note the age, type, and reason of the amputation; info about the use of prostheses (cosmetic, body-powered, self-powered, etc.) along with the (dis)advantages and consequences of their usage; type and degree of phantom limb sensation and pain. Moreover, we are still improving the clinical data list in order to include more information about the anatomical features of the subjects. Two pictures of the stump are taken. Data are stored anonymously for a limited amount of time and they are available for deletion upon request of the subject.

2) Stimulus: A set of hand and wrist movements of interest was defined, inspired by standard lists of hand postures and grasps, grasping taxonomies, and reports of similar experiments (see, e.g., [30]-[34]), as well as the Disabilities of the Arm, Shoulder and Hand protocol for functional movements [35]. A total of 52 movements were selected, divided into four main classes:

- 12 basic movements of the fingers (flexions and extensions);

- 8 isometric, isotonic hand configurations ("hand postures");

- 9 basic movements of the wrist (adduction/abduction, flexion/extension and pronation/supination); and

- 23 grasping and functional movements - in this case, everyday objects are presented to the subject for grasping, in order to mimick a daily-life action.

Figure 2 graphically shows each movement; synthetic text descriptions and related references (if any) can be found in Table I.

3) Data Acquisition: The subject sits comfortably on an adjustable chair, in front of a table with a large screen. The sEMG electrodes, dataglove and inclinometer are worn on the right hand. The subjects are presented with short movies appearing on the screen and are asked to simply replicate the
TABLE I

SYNTHETIC DESCRIPTIONS OF THE 52 MOVEMENTS OF INTEREST, ALONG WITH A REFERENCE, IF AVAILABLE.

\begin{tabular}{|c|c|c|c|}
\hline & \# & Description & Ref. \\
\hline \multirow{6}{*}{$\begin{array}{l}\dot{\Delta} \\
\vec{z} \\
\dot{\Delta} \\
\dot{\Delta} \\
\stackrel{\Delta}{\Xi}\end{array}$} & $1-2$ & Index flexion and extension & [13] \\
\hline & $3-4$ & Middle flexion and extension & [13] \\
\hline & $5-6$ & Ring flexion and extension & [13] \\
\hline & $7-8$ & Little finger flexion and extension & [13] \\
\hline & $9-10$ & Thumb adduction and abduction & [13] \\
\hline & $11-12$ & Thumb flexion and extension & \\
\hline \multirow{8}{*}{ 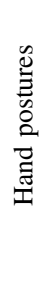 } & 1 & Thumb up & [36] \\
\hline & 2 & $\begin{array}{l}\text { Flexion of ring and little finger; thumb flexed } \\
\text { over middle and little }\end{array}$ & \\
\hline & 3 & Flexion of ring and little finger & [25] \\
\hline & 4 & Thumb opposiing base of little finger & [25] \\
\hline & 5 & Abduction of the fingers & [25] \\
\hline & 6 & Fingers flexed together & [25] \\
\hline & 7 & Pointing index & [4] \\
\hline & 8 & Fingers closed together & [5] \\
\hline \multirow{5}{*}{ 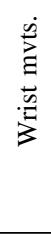 } & $1-2$ & $\begin{array}{l}\text { Wrist supination and pronation (rotation axis } \\
\text { through the middle finger) }\end{array}$ & [36] \\
\hline & $3-4$ & $\begin{array}{l}\text { Wrist supination and pronation (rotation axis } \\
\text { through the little finger) }\end{array}$ & \\
\hline & $5-6$ & Wrist flexion and extension & [36] \\
\hline & $7-8$ & Wrist radial and ulnar deviation & [5] \\
\hline & 9 & Wrist extension with closed hand & \\
\hline \multirow{18}{*}{ 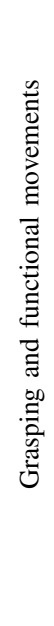 } & $1-2$ & Large and small diameter & [30] \\
\hline & 3 & Fixed hook & {$[30]$} \\
\hline & 4 & Index finger extension & [30] \\
\hline & 5 & Medium wrap & [30] \\
\hline & 6 & Ring & [30] \\
\hline & 7 & Prismatic four fingers & {$[30]$} \\
\hline & 8 & Stick & [30] \\
\hline & 9 & Writing tripod & {$[30]$} \\
\hline & $10-12$ & Power, three finger, and precision sphere & [30] \\
\hline & 13 & Tripod & [30] \\
\hline & $14-15$ & Prismatic and tip pinch & [30] \\
\hline & 16 & Quadpod & [30] \\
\hline & 17 & Lateral & [30] \\
\hline & $18-19$ & Parallel extension and flexion & [30] \\
\hline & 20 & Power disk & [30] \\
\hline & 21 & Open a bottle with a tripod grasp & [34] \\
\hline & 22 & $\begin{array}{l}\text { Turn a screw (grasp the screwdriver with a } \\
\text { stick grasp (8)) }\end{array}$ & \\
\hline & 23 & $\begin{array}{l}\text { Cut something (grasp the knife with an index } \\
\text { finger extension grasp (4)) }\end{array}$ & [34] \\
\hline
\end{tabular}

movements depicted in the movies as accurately as possible. Amputees are handled as follows: the sEMG electrodes are worn on the stump while the dataglove and the inclinometer are worn on the intact limb. The subject is asked to bilaterally perform the movement shown on the screen. (This procedure can be seen as fusion of bilateral and imitation training, see [3].) Each subject first undergoes a "training phase" to get familiar with the procedure, during which each movement of the first three classes and three movements of the fourth class are repeated three times (no data are recorded). After the training phase, a sequential series of ten repetitions of each class of movements is presented to the subject while data are recorded. Each movie lasts five seconds and three seconds of rest are allowed in-between movements. The four movement classes are functionally grouped into three "exercises" (the second and third classes are grouped into the second exercise). Notice that the sequences are intentionally not 


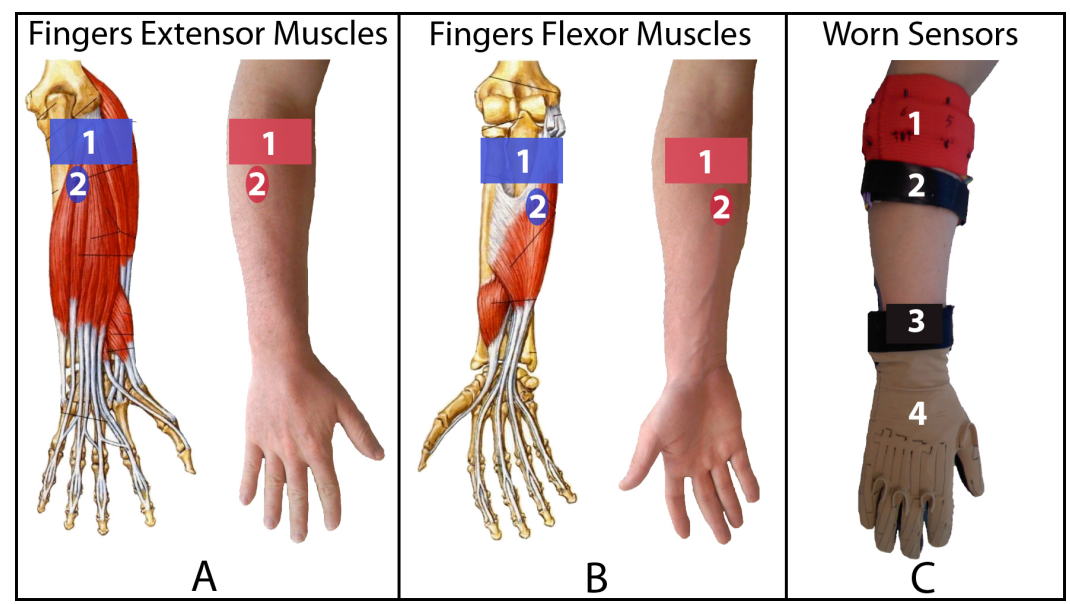

Fig. 1. Placement of the electrodes: A. sEMG electrodes placed on finger extensor muscles (A.1 Equally spaced electrodes; A.2 Spare electrode); B. sEMG electrodes placed on finger flexor muscles (B.1 Equally spaced electrodes; B.2 Spare electrode); C. all the sensors positioned on the arm (C.1 Equally spaced electrodes; C.2 Spare electrode; C.3 Inclinometer; C.4 Cyberglove II);

randomized in order to induce repetitive, almost unconscious movements. The three exercises are completed in 16,23 , and 31 minutes. In order to avoid muscle fatigue and its influence on the sEMG signal, 5 minutes of rest are allowed between the training sequence and the first exercise and between each exercise and the following one. In total, the experiment lasts about 100 minutes.

4) Interconnection and Data Synchronization: The dataglove streams data through a Bluetooth-tunneled serial port at a rate of $25 \mathrm{~Hz}$; the inclinometer and sEMG electrodes are connected to a standard DAQ card sampling the signals at $100 \mathrm{~Hz}$. Both the dataglove and the DAQ card are connected to a laptop equipped with Windows XP and custom software developed for this study. In order to give maximum data processing flexibility to the NINAPRO database user, we store the data totally asynchronously, tagging each sample with an accurate timestamp. Accurate timestamping on Windows is enforced by the HRT library [37], whose precision is of the order of magnitude of the microsecond. The stimulus generator is then used to generate the labels for each sample, as a further data stream running at $25 \mathrm{~Hz}$; each sample can therefore be associated with a subject, exercise number, and movement index. Notice however, that the database does not rely on how accurately the subjects follow the stimulus, since the ground truth is collected using the dataglove and the inclinometer.

5) The Database: The current (first) version of the database [38] contains data from 27 intact subjects (20M/7F, $25 / 2$ right-/left-handed, age $28 \pm 3.4 \mathrm{yrs}$ ). For each subject, three data files are stored in plain ASCII format, containing in turn the electrodes and inclinometer data, the Cyberglove data, and the stimulus data. The data stored are the ones coming from the electrodes, the inclinometer and the cyberglove without any pre-processing. Each datum is arranged in a row, consisting of a timestamp plus the sensor values. Five pictures are also stored for each subject, three previews of the data of each exercise plus two pictures of the forearm and of the hand with and without the acquisition setup. A record of the identity of all the subjects is kept, although these private details are explicitly excluded from publication and analysis.

\section{ANALYSIS}

A primary motivation for constructing the NINAPRO database is to drastically increase the number of considered subjects and movements with respect to prior work. In this section, the database is used to investigate experimentally whether this increase has a detrimental effect on the classification accuracy.

We consider three sets of movements of increasing size, namely: the three grasp movements considered in [28], eleven of the twelve movements considered in $[13]^{4}$, and all 52 movements contained in the NINAPRO database. As a first experiment, we perform a qualitative analysis of the data by visualizing the sEMG signals, where in addition to the three posture cases we also experiment with an increasing number of subjects (i.e., 1, 8 and 27 subjects). Subsequently, we estimate the performance on the task of movement classification to establish quantitative results.

\section{A. Preprocessing}

All data are synchronized by linearly interpolating all data to the highest recording frequency (i.e., $100 \mathrm{~Hz}$ ). Both sEMG and Cyberglove signals are subsequently low-pass filtered at $1 \mathrm{~Hz}$ using a zero-phase second order Butterworth filter. Samples with an ambiguous label, that is those recorded during transition between rest and the actual movement, are removed by dividing each movement (including rest) in three equally sized segments and only retaining data from the center segment. To ensure computational feasibility, we then average the data contained in this center segment to obtain a single sample per movement. Note that there are

\footnotetext{
${ }^{4}$ The grouped extension movement of the middle, ring, and little finger is omitted from the analysis due to lack of a suitable alternative in our database, whereas for the corresponding flexion movement we instead consider flexion of just the ring and little finger (cf. Table I).
} 

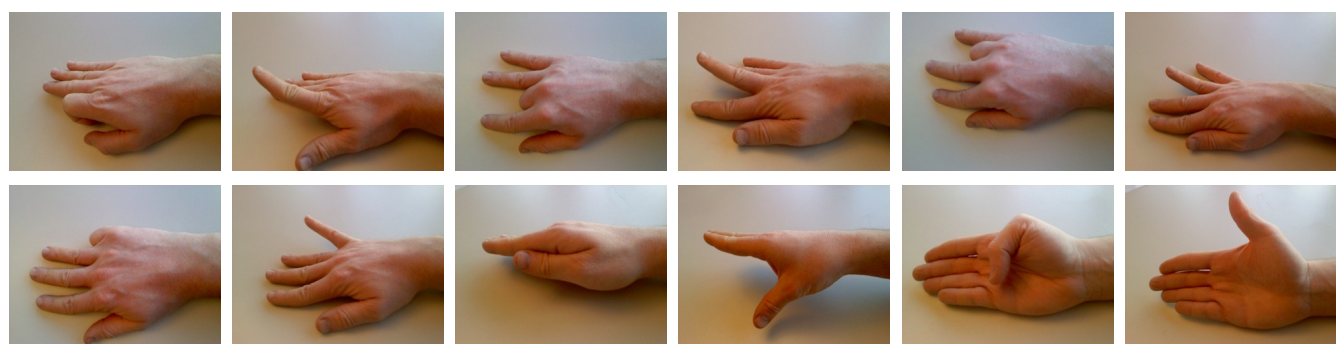

(a) Basic movements of the fingers (flexions and extensions).
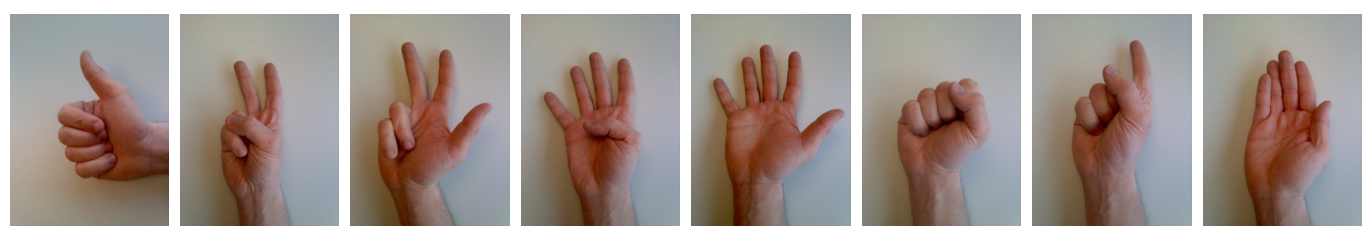

(b) Isometric, isotonic hand configurations ("hand postures").
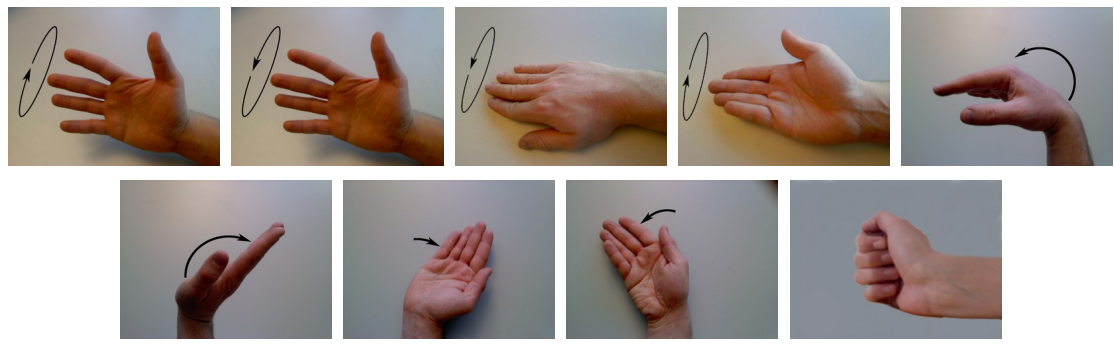

(c) Basic movements of the wrist.
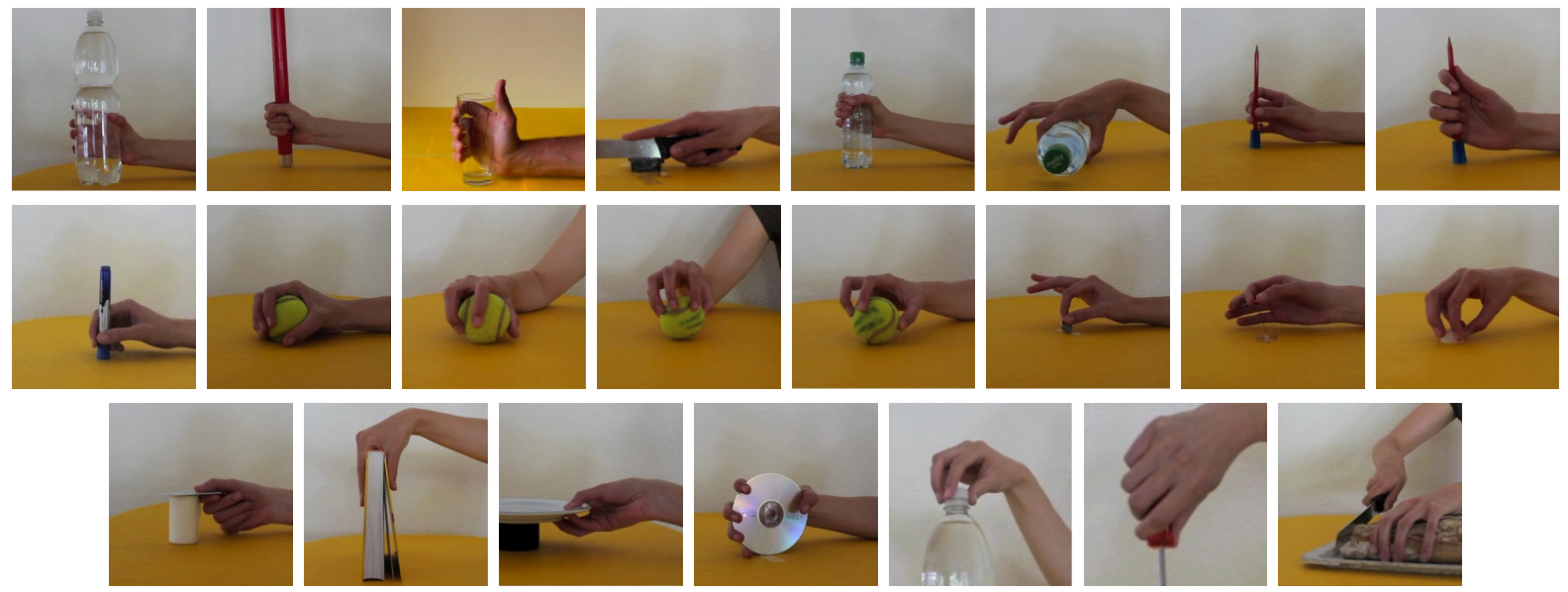

(d) Grasping and functional movements.

Fig. 2. The 52 movements of interest.

thus ten samples for each subject-movement combination (i.e., one per repetition) and that half of the samples belong to the rest movement. The described preprocessing chain is illustrated in Figure 3. Finally, the data for individual subjects is normalized such that each of the sEMG signals has zero mean and unit standard deviation.

\section{B. Visualization}

In order to give insight into the relation between movements and sEMG signals, we visualize the latter using principal component analysis (PCA). Figure 4 demonstrates the two principal components for each of the nine cases considered. In case of a single subject and three movements (cf. top left panel), it is relatively easy to distinguish the movements, since they occupy separated regions in the PCA space with minimal overlap. This overlap increases drastically, however, when combining data from multiple subjects, most prominently so for the complete set of 27 subjects. This is a clear indication of high variability in the recorded sEMG signals among subjects.

Increasing the number of movements has a similar detrimental effect on the separation of movements. The small, medium, and complete set of movements are displayed along the columns in Figure 4. Even when restricting ourselves 


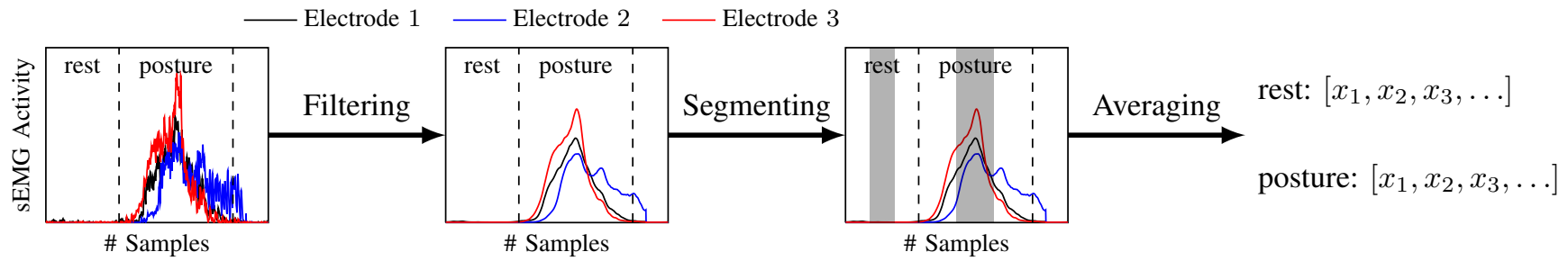

Fig. 3. Illustration of the preprocessing chain consisting of filtering, segmenting, and averaging. The gray shaded area indicates the center segment over which the sEMG signal is subsequently averaged. For clarity reasons, the figure displays only three out of ten electrodes.

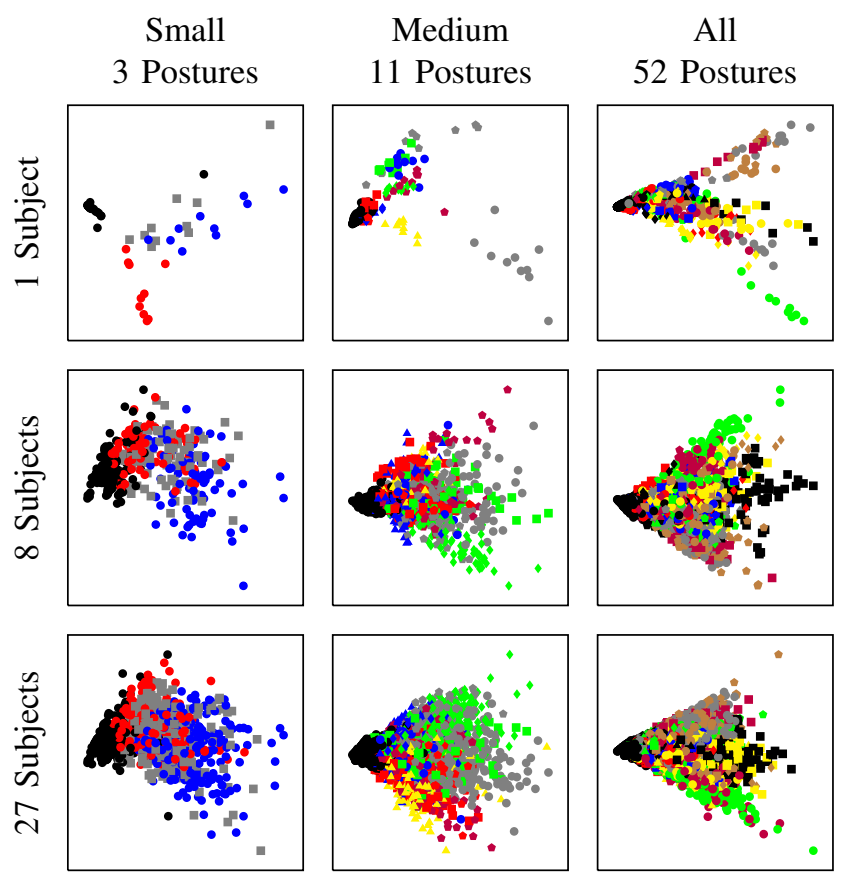

Fig. 4. Two principal components of the sEMG signals with an increasing number of subjects and movements. Movement classes are indicated by a combination of marker shape and color. This figure is therefore best viewed in color.

to a single subject (top row), it is clear that separability deteriorates when increasing the number of movements. In the relatively mild medium setting we still observe that the movements occupy distinct regions in the two principal components of the sEMG space. Moving to the complete case of 52 movements, however, it becomes very difficult to distinguish movements. Not surprisingly, the problem of (visually) separating movements is even more challenging when combining data from multiple subjects with this large number of movements.

\section{Classification}

Visualization of movements using PCA provides some insight into the expected difficulty of classification with a large number of movements. Ultimately, however, we are interested in a quantitative estimate of the classification performance. To this extent, we employ a Least-Squares support vector machine (LS-SVM, see [39]) classifier to predict the movement class in the three previously described settings. Similar to the more popular standard support vector machine (SVM), LS-SVM is a kernel-based classifier that attempts to maximize the margin between two classes. Advantages of LS-SVM over SVM, however, are that multiple classifiers in the one-vs-all multi-class scheme can be learned concurrently ${ }^{5}$ and that the leave-one-out error can be computed in closed form after training only once on all training samples [40]. Moreover, it was demonstrated experimentally that the classification performance is typically comparable [41].

In our experiments, a multi-class LS-SVM with RBF kernel is trained for each distinct subject utilizing five repetitions per movement, while the remaining five repetitions are used for testing. The number of classes is equal to the reported number of movements plus the rest movement. Furthermore, note that the amount of train and test data is proportional to the number of classes, in order to ensure a fair comparison between settings with a different number of movements. Hyperparameters are optimized using grid search, where the trade-off parameter $C \in\left\{2^{-2}, 2^{-1}, \ldots, 2^{13}, 2^{14}\right\}$ and kernel bandwidth $\gamma \in\left\{2^{-12}, 2^{-11}, \ldots, 2^{6}, 2^{7}\right\}$. In total, the same experiment is repeated 25 times, where each trial is a random split over the ten repetitions available for each movement.

Figure 5 demonstrates the balanced classification error averaged over all 27 subjects and 25 trials when considering the small, medium, and complete set of movements. The reported errors range roughly from $10 \%$ to $20 \%$ and are thus comparable to those reported in related work (considering the number of movements), thereby confirming the correctness of the experimental protocol and data. However, as predicted during the qualitative analysis, we indeed observe that performance deteriorates when increasing the number of movements. While the small case results in a classification error of $7.5 \pm 7.2 \%$, this increases to $20.3 \pm 6.9 \%$ when considering all movements. Moreover, the standard deviation of the classification errors is relatively high, indicating that there is considerable variability in the classification performance among different subjects. It follows that a large number of subjects is required to reliably estimate performance on this type of classification tasks.

Besides the standard approach of both training and testing the model on data from a single subject, we also evaluate each model on the test data of the remaining subjects. These results should give an impression about the extent to which

\footnotetext{
${ }^{5}$ Assuming identical hyperparameter configurations for all classifiers.
} 


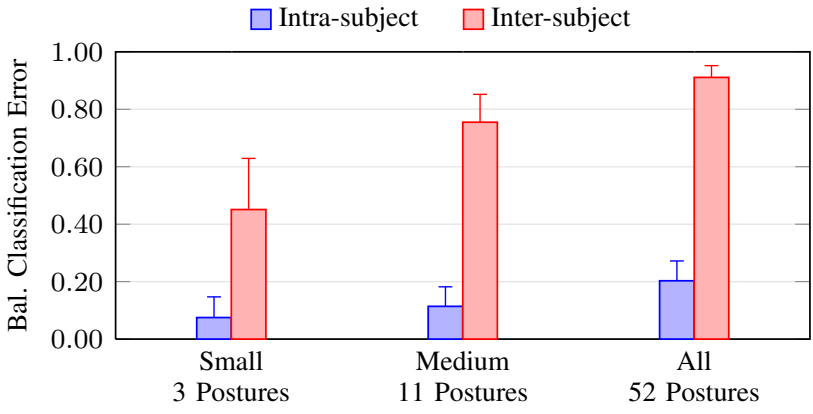

Fig. 5. Balanced classification error for the small, medium, and complete set of movements when train and test data belong to the same subject (cf. "intra-subject") and when the test subject is different from the train subject (cf. "inter-subject"). All results report the mean and unit standard deviation over the 27 subjects and over 25 random splits.

previously trained models can be employed for new subjects. Unfortunately, the inter-subject classification performance is inferior in all settings and only marginally above chance level (i.e., $1-\frac{1}{c}$ with $c$ being the number of classes). In other words, the data for a given subject are not very representative for the data of other subjects. This confirms our earlier observation that there is considerable inter-subject variability, and a more advanced methodology is required to successfully transfer models to new subjects.

\section{CONCLUSIONS}

In this paper we have introduced the first version of the NINAPRO database, a tool for the biorobotics community, especially for those concerned with myoelectric control of advanced hand prostheses. We have described the acquisition setup and experimental protocol, which stem from existing literature; then the stimulus, consisting of a very large set of finger, hand and wrist movements, distilled by various preexisting sources; lastly, we have described the very building of the first version of the database, obtained from 27 intact subjects.

Preliminary analysis, carried out by applying a standard machine learning method (Least-Squares SVMs) to the database after performing standard data preprocessing, reveals that the data are perfectly usable and the classification error rates are comparable to those found in literature (e.g., [3]), given the same conditions, i.e., considering the same number of movements and subjects. This acts as a backvalidation of the acquisition setup, the experimental protocol and the data storage.

The results of the analysis also clearly indicate that considering more movements (classes) and more subjects cause a dramatic increase in the error rates; and that there is significant inter-subject variability, as the inter-subject classification accuracy approaches chance level. These observations justify our aim of constructing a publicly available database from a large number of subjects performing a large number of movements.

\section{Future Work}

The NINAPRO database is an ongoing work aiming at improving the state-of-the-art in sEMG control of AHPs along three dimensions, namely (1) establishing a standard, widely accepted benchmark; (2) collecting a very high number of hand postures, and (3) from a very high number of subjects, both intact and amputated.

As far as item (1) and (2) are concerned, we think that an interesting path to pursue is to add dynamic finger/hand/wrist data to the NINAPRO database, in order to study force- or impedance-controlled hand prostheses. This will be enforced in the further releases of the database thanks to the use of a custom-built force-sensing device, able to record fingertip forces both during flexion and extension of the fingers, plus thumb adduction and abduction.

As far as item (3) is concerned, further releases of the database will contain data recorded from more subjects, both intact and especially amputated. Amputees will be recruited and examined thanks to our clinical partner. The setup is portable, which greatly increases the range across which we will be able to find volunteers. Recording the diversity of the amputations will be a key issue for the scientific value of the NINAPRO database, as the researchers will be able to associate the characteristic of the amputee to the best matching methods of analysis and control.

Lastly, as the final version of the database will be released, novel machine learning methods will have to be devised and applied to it, as it is clear that it will constitute a real challenge for the state of the art.

\section{ACKNOWLEDGEMENTS}

We thank Mr. Ramviyas Parasuraman for early work on the acquisition software. This work is partially supported by the Swiss National Science Foundation Sinergia project NinAPRO (Non-Invasive Adaptive Prosthetics).

\section{REFERENCES}

[1] D. J. Atkins, "Epidemiologic overview of individuals with upper-limb loss and their reported research priorities," Journal of Prosthetics \& Orthotics, vol. 8, no. 1, pp. 2-11, 1996.

[2] O. F. T. Tsuji, M. Kaneko, and A. Otsuka, "A human-assisting manipulator teleoperated by EMG signals and arm motions," IEEE Transactions on Robotics and Automation, vol. 19, no. 2, pp. 210222, apr 2003.

[3] C. Castellini, E. Gruppioni, A. Davalli, and G. Sandini, "Fine detection of grasp force and posture by amputees via surface electromyography," Journal of Physiology (Paris), vol. 103, no. 3-5, pp. 255-262, 2009.

[4] T. R. Farrell and R. F. Weir, "A comparison of the effects of electrode implantation and targeting on pattern classification accuracy for prosthesis control," IEEE Transactions on Biomedical Engineering, vol. 55, pp. 2198-2211, March 2008.

[5] B. Crawford, K. Miller, P. Shenoy, and R. Rao, "Real-time classification of electromyographic signals for robotic control," in Proceedings of AAAI, 2005, pp. 523-528.

[6] E. Engeberg and S. Meek, "Improved grasp force sensitivity for prosthetic hands through force-derivative feedback," IEEE Transactions on Biomedical Engineering, vol. 55, no. 2, pp. 817-821, 2008.

[7] E. Engeberg, S. Meek, and M. Minor, "Hybrid force-velocity sliding mode control of a prosthetic hand," IEEE Transactions on Biomedical Engineering, vol. 55, no. 5, pp. 1572-1581, 2008

[8] H. Müller, J. Kalpathy-Cramer, I. Eggel, S. Bedrick, R. Said, B. Bakke, C. E. J. Kahn, and W. Hersh, "Overview of the clef 2009 medical image retrieval track," in Working Notes of CLEF 2009, Corfu, Greece, September 2009 . 
[9] M. Everingham, L. Van Gool, C. K. I. Williams, J. Winn, and A. Zisserman, "The PASCAL Visual Object Classes Challenge 2011 (VOC2011) Results," http://www.pascalnetwork.org/challenges/VOC/voc2011/workshop/index.html.

[10] M. Zecca, S. Micera, M. C. Carrozza, and P. Dario, "Control of multifunctional prosthetic hands by processing the electromyographic signal," Critical Reviews in Biomedical Engineering, vol. 30, no. 4-6, pp. 459-485, 2002.

[11] J. L. G. Nielsen, S. Holmgaard, N. Jiang, K. B. Englehart, D. Farina, and P. A. Parker, "Simultaneous and proportional force estimation for multifunction myoelectric prostheses using mirrored bilateral training," IEEE Transactions on Biomedical Engineering, vol. 58, no. 3, pp. 681-688, 2011.

[12] S. Bitzer and P. van der Smagt, "Learning EMG control of a robotic hand: Towards active prostheses," in Proceedings of ICRA, International Conference on Robotics and Automation, Orlando, Florida, USA, may 2006, pp. 2819-2823.

[13] F. V. Tenore, A. Ramos, A. Fahmy, S. Acharya, R. Etienne-Cummings, and N. V. Thakor, "Decoding of individuated finger movements using surface electromyography," IEEE Transactions on Biomedical Engineering, vol. 56, no. 5, pp. 1427-1434, 2009.

[14] P. K. Artemiadis and K. J. Kyriakopoulos, "EMG-based control of a robot arm using low-dimensional embeddings," IEEE Transactions on Robotics, vol. 26, no. 2, pp. 393-398, 2009.

[15] — _ "An emg-based robot control scheme robust to time-varying EMG signal features," IEEE Transactions on Information Technology in Biomedicine, vol. 14, no. 3, pp. 582-588, 2010.

[16] _ - "A switching regime model for the emg-based control of a robot arm," IEEE Transactions on Systems, Man and Cybernetics Part B: Cybernetics, vol. 41, no. 1, pp. 53-63, 2011.

[17] J. Vogel, C. Castellini, and P. van der Smagt, "EMG-based teleoperation and manipulation with the DLR LWR-III," in Proceedings of IROS - International Conference on Intelligent Robots and Systems, 2011, pp. 672-678.

[18] F. R. Finley and R. W. Wirta, "Myocoder studies of multiple myopotential response," Archives of Physical Medicine and Rehabilitation, vol. 48 , no. 11 , pp. $598-601,1967$

[19] S. Micera, J. Carpaneto, and S. Raspopovic, "Control of hand prostheses using peripheral information," IEEE Reviews in Biomedical Engineering, vol. 3, pp. 48-68, Oct. 2010.

[20] B. Peerdeman, D. Boere, H. Witteveen, R. Huis in 't Veld, H. Hermens, S. Stramigioli, H. Rietman, P. Veltink, and S. Misra, "Myoelectric forearm prostheses: State of the art from a user-centered perspective," Journal of Rehabilitation Research and Development, vol. 48, no. 6, pp. 719-738, 2011.

[21] U. Kuruganti, H. Daley, and K. B. Englehart, "High density emg data of normally limbed and transradial amputees," in Proceedings of the Congress of the International Society of Electrophysiology and Kinesiology, Aalborg, Denmark, 2010.

[22] R. Merletti, M. Aventaggiato, A. Botter, A. Holobar, H. Marateb, and T. Vieira, "Advances in surface EMG: Recent progress in detection and processing techniques," Critical reviews in biomedical engineering, vol. 38, no. 4, pp. 305-345, 2011.

[23] R. Merletti, A. Botter, A. Troiano, E. Merlo, and M. Minetto, "Technology and instrumentation for detection and conditioning of the surface electromyographic signal: State of the art," Clinical Biomechanics, vol. 24, pp. 122-134, 2009.

[24] C. J. De Luca, "The use of surface electromyography in biomechanics," Journal of Applied Biomechanics, vol. 13, no. 2, pp. 135-163, 1997.
[25] F. C. P. Sebelius, B. N. Rosen, and G. N. Lundborg, "Refined myoelectric control in below-elbow amputees using artificial neural networks and a data glove," Journal of Hand Surgery, vol. 30, no. 4, pp. 780-789, Jul 2005.

[26] C. Cipriani, C. Antfolk, M. Controzzi, G. Lundborg, B. Rosen, M. Carrozza, and F. Sebelius, "Online myoelectric control of a dexterous hand prosthesis by transradial amputees," IEEE Transactions on Neural Systems and Rehabilitation Engineering, vol. 19, no. 3, pp. 260-270, june 2011.

[27] C. Castellini and P. van der Smagt, "Surface EMG in advanced hand prosthetics," Biological Cybernetics, vol. 100, no. 1, pp. 35-47, 2009.

[28] C. Castellini, A. E. Fiorilla, and G. Sandini, "Multi-subject / dailylife activity EMG-based control of mechanical hands," Journal of Neuroengineering and Rehabilitation, vol. 6, no. 41, 2009.

[29] H. Tsuji, H. Ichinobe, K. Ito, and M. Nagamachi, "Discrimination of forearm motions from emg signals by error back propagation typed neural network using entropy," IEEE Transactions, Society of Instrument and Control Engineers, vol. 29, no. 10, pp. 1213-1220, 1993.

[30] T. Feix, "Grasp taxonomy comparison," Otto Bock GmbH, Tech. Rep., 2008. [Online]. Available: http://grasp.xief.net

[31] M. Cutkosky, "On grasp choice, grasp models, and the design of hands for manufacturing tasks," IEEE Transactions on Robotics and Automation, vol. 5, no. 3, pp. 269 -279, Jun 1989.

[32] N. Kamakura, M. Matsuo, H. Ishii, F. Mitsuboshi, and Y. Miura, "Patterns of static prehension in normal hands," The American journal of occupational therapy: official publication of the American Occupational Therapy Association, vol. 34, no. 7, pp. 437-445, 1980.

[33] S. J. Edwards, D. J. Buckland, and J. D. McCoy-Powlen, Developmental and Functional Hand Grasps. Slack Incorporated, 2002.

[34] P. L. Hudak, P. C. Amadio, and C. Bombardier, "Development of an upper extremity outcome measure: the dash (disabilities of the arm, shoulder and hand) the upper extremity collaborative group (uecg)," American Journal of Industrial Medicine, vol. 29, no. 6, pp. 602-8, 1996.

[35] W. Hersh, H. Müller, J. Kalpathy-Cramer, and E. Kim, "The imageclefmed medical image retrieval task test collection," in Society for Imaging Informatics in Medicine (SIIM) 2008, Seattle, USA, May 2008.

[36] R. Kato, H. Yokoi, and T. Arai, "Competitive learning method for robust emg-to-motion classifier," in Proceedings Intelligent Autonomus Systems, 2006, pp. 946-953.

[37] J. Nilsson, "Implementing a continuously updating, high-resolution time provider for windows," The MSDN Magazine, 2004. [Online] Available: http://msdn.microsoft.com/en-us/magazine/cc163996.aspx

[38] F. Chabbey, "Data acquisitions for electromyograms," Bachelor's Thesis, Department of Business Information Systems at the University of Applied Sciences Western Switzerland (HES- SO Valais), Sierre Switzerland, 2011.

[39] J. A. K. Suykens, T. V. Gestel, J. D. Brabanter, B. D. Moor, and J. Vandewalle, Least Squares Support Vector Machines. Singapore: World Scientific Publishing Co., Pte, Ltd., 2002.

[40] G. C. Cawley, "Leave-one-out cross-validation based model selection criteria for weighted 1s-svms," in IJCNN-2006: Proceedings of the International Joint Conference on Neural Networks, Vancouver, BC, Canada, July 2006, pp. 1661-1668.

[41] T. V. Gestel, J. A. K. Suykens, B. Baesens, S. Viaene, J. Vanthienen, G. Dedene, B. D. Moor, and J. Vandewalle, "Benchmarking least squares support vector machine classifiers," Machine Learning, vol. 54, pp. 5-32, January 2004. 